\title{
Configuration localized Morse wave functions: Application to vibrational transitions in anharmonic diatomic molecules
}

\author{
M. Carvajal, J. M. Arias, and J. Gómez-Camacho \\ Departamento de Física Atómica, Molecular y Nuclear, Facultad de Física, Universidad de Sevilla, Apartado 1065, 41080 Sevilla, Spain
}

(Received 16 September 1998)

\begin{abstract}
The bound states of a Morse potential are described in terms of a basis of states that are characterized for being eigenstates of the Morse variable. These states are strongly localized in configuration space; thus they are called configuration localized Morse (CLM) wave functions. These are shown to provide a powerful tool to calculate analytically, to a good approximation, matrix elements of arbitrary functions of the interatomic separation between vibrational states of anharmonic diatomic molecules. Applications of CLM wave functions to the calculation of vibrational transitions in $\mathrm{HF}$ and $\mathrm{DF}$ diatomic molecules are presented. [S1050-2947(99)10402-5]
\end{abstract}

PACS number(s): 31.15.Ar, 31.15.Hz, 03.65.Ca, 33.20.Tp

\section{INTRODUCTION}

Since its introduction in 1929 [1], the Morse potential has been a useful tool for representing more realistic potentials in different branches of physics. It is a two-parameter anharmonic potential that allows for the complete analytical solution of the Schrödinger equation, hence providing wave functions for the eigenstates. They can be written in compact form by using the generalized Laguerre polynomials. However, the use of these functions to calculate matrix elements of relevant operators is cumbersome and one has to be extremely careful, especially when treating states high in excitation energy, since the corresponding wave functions are rapidly oscillating functions and their integration can produce numerical errors. Several methods have been published in the past to deal with this problem [2-6].

The Morse potential has been thoroughly used to model the interatomic interaction in the field of molecular physics. Following the introduction of algebraic models in molecular spectroscopy the Morse potential has become even more popular since the knowledge of the existence of an isomorphism between it and the Lie algebra su(2) [7]. This isomorphism allows for a connection between the algebraic treatment and the description in configuration space.

In this paper we introduce a basis of configuration localized Morse (CLM) states. These are particular combinations of bound Morse eigenstates that localize the system in configuration space. In that basis, closed expressions for the matrix elements of different operators of interest can be obtained. By using CLM wave functions good approximations to the matrix elements of arbitrary operators between Morse eigenstates are obtained.

The paper is structured as follows: In Sec. II, we revise the analytic solution of the Schrödinger equation with a Morse potential to present consistently the notation. In Sec. III the CLM states are introduced formally. Their properties are discussed and used to evaluate matrix elements of different operators. In Sec. IV the formalism presented in the previous section is applied to the diatomic molecules $\mathrm{HF}$ and DF. In Sec. $\mathrm{V}$ we outline some further applications of the CLM states. Finally, the summary and conclusions are presented in Sec. VI.

\section{REVISITING THE MORSE POTENTIAL}

The Morse potential can be written as

$$
V(x)=D\left[(1-\exp [-x])^{2}-1\right],
$$

where $-D$ is the value of the potential in its minimum and $x=\beta\left(r-r_{e}\right)$ is related to the separation from the equilibrium position $\left(r_{e}\right)$. The parameter $\beta$ is the inverse of the range of the potential. As mentioned in the Introduction, the Morse Hamiltonian can be related to the su(2) algebra. Thus, its bound states can be labeled by $|j m\rangle$. The quantum number $j$ is an integer related to the depth of the potential through

$$
D=\frac{\hbar^{2} \beta^{2}}{2 \mu}\left(j+\frac{1}{2}\right)^{2}
$$

where $\mu$ is the reduced mass of the system. A potential characterized by a value of $D$ supports $j$ bound states plus one state with zero energy. This last state is not normalizable and, since we are treating only bound states, it will not be considered in the rest of this paper. The label $m$ is related to the binding energy of the state,

$$
\boldsymbol{\epsilon}_{m}=-\frac{\hbar^{2} \beta^{2}}{2 \mu} m^{2}, \quad(m=1,2, \ldots, j) .
$$

Thus, the state more tightly bound corresponds to $m=j$. As is usual in molecular physics we are introducing the alternative quantum number $v=j-m$, which corresponds to the number of anharmonic phonons. Consequently $v$ runs from 0 to $j-1$ and the ground state has $v=0$. For a diatomic molecule the Morse parameter $\beta$ and the $j$ quantum number can be obtained from the usual spectroscopic harmonic $\left(\omega_{e}\right)$ and anharmonic $\left(\omega_{e} x_{e}\right)$ constants through

$$
\beta^{2}=\frac{2 \mu}{\hbar^{2}} \omega_{e} x_{e}, \quad\left(j+\frac{1}{2}\right)^{2}=\frac{2 \mu D}{\hbar^{2} \beta^{2}}=\frac{1}{4}\left(\frac{\omega_{e}}{\omega_{e} x_{e}}\right)^{2}
$$

With this notation the bound eigenstates of the Morse Hamiltonian are written as 


$$
\langle x \mid j v\rangle=\Psi_{j v}(x)=\mathcal{N}_{j v}^{-1 / 2} \exp \left[-\frac{y}{2}\right] y^{j-v} L_{v}^{(2 j-2 v)}(y),
$$

where $\mathcal{N}_{j v}$ is a normalization constant, $y=(2 j+1) \exp [-x]$ is the Morse variable, and $L_{s}^{(p)}(y)$ are the generalized Laguerre polynomials of degree $s$ and order $p$. For later purposes, it is useful to introduce a polynomial of degree $j-1$ defined by

$$
P_{j-1}^{(v)}(y)=y^{j-v-1} L_{v}^{(2 j-2 v)}(y) .
$$

These polynomials satisfy the orthogonality condition,

$$
\int_{0}^{\infty} d y y \exp [-y] P_{j-1}^{(v)}(y) P_{j-1}^{\left(v^{\prime}\right)}(y)=\mathcal{N}_{j v} \delta_{v, v^{\prime}},
$$

derived from the orthogonality condition for the Morse wave functions. At this point it is interesting to note that the generalized Laguerre polynomials of order $1, L_{n}^{(1)}(y)$, satisfy the orthogonality condition

$$
\int_{0}^{\infty} d y y \exp [-y] L_{n}^{(1)}(y) L_{n^{\prime}}^{(1)}(y)=(n+1) \delta_{n, n^{\prime}} .
$$

Comparing the last two equations, it is clear that there is an orthonormal transformation from the polynomials $\left\{\mathcal{N}_{j v}^{-1 / 2} P_{j-1}^{(v)}(y) ; v=0,1, \ldots, j-1\right\}$ to the generalized Laguerre polynomials of order $1\left\{(n+1)^{-1 / 2} L_{n}^{(1)}(y) ; n\right.$ $=0,1, \ldots, j-1\}$, which allows us to write

$$
\sum_{v=0}^{j-1} \mathcal{N}_{j v}^{-1} P_{j-1}^{(v)}(y) P_{j-1}^{(v)}(z)=\sum_{n=0}^{j-1}(n+1)^{-1} L_{n}^{(1)}(y) L_{n}^{(1)}(z) \text {. }
$$

Combining this last expression with the Christoffel-Darboux formula [8], it is obtained that

$$
\begin{aligned}
\sum_{v=0}^{j-1} & \mathcal{N}_{j v}^{-1} P_{j-1}^{(v)}(y) P_{j-1}^{(v)}(z) \\
& =\sum_{n=0}^{j-1}(n+1)^{-1} L_{n}^{(1)}(y) L_{n}^{(1)}(z) \\
& =-\frac{L_{j}^{(1)}(y) L_{j-1}^{(1)}(z)-L_{j-1}^{(1)}(y) L_{j}^{(1)}(z)}{y-z} .
\end{aligned}
$$

Let us consider now the case in which $y$ and $z$ are zeros of $L_{j}^{(1)}(y)$; they will be denoted by $y_{k}$ and $y_{\ell}$. There are $j$ such zeros. For the case in which $y_{k} \neq y_{\ell}$, Eq. (10) gives

$$
\sum_{v=0}^{j-1} \mathcal{N}_{j v}^{-1} P_{j-1}^{(v)}\left(y_{k}\right) P_{j-1}^{(v)}\left(y_{\ell}\right)=0 \text {. }
$$

For the case in which $y_{k}=y_{\ell}$, taking the limit when $z$ $\rightarrow y_{k}$, it is obtained that

$$
\sum_{v=0}^{j-1} \mathcal{N}_{j v}^{-1} P_{j-1}^{(v)}\left(y_{k}\right) P_{j-1}^{(v)}\left(y_{k}\right)=N_{k}
$$

where

$$
N_{k}=\frac{j+1}{y_{k}}\left[L_{j+1}^{(1)}\left(y_{k}\right)\right]^{2}=\frac{j+1}{y_{k}}\left[L_{j-1}^{(1)}\left(y_{k}\right)\right]^{2} .
$$

In deriving these expressions we have used the following known relations for the generalized Laguerre polynomials, evaluated at the zeros of $L_{j}^{(1)}(y)$ :

$$
\begin{gathered}
L_{j-1}^{(1)}\left(y_{k}\right)=-L_{j+1}^{(1)}\left(y_{k}\right), \\
{\left[\frac{d}{d y} L_{j}^{(1)}(y)\right]_{y_{k}}=-\frac{j+1}{y_{k}} L_{j-1}^{(1)}\left(y_{k}\right) .}
\end{gathered}
$$

\section{CONFIGURATION LOCALIZED MORSE (CLM) STATES}

From the $(j-1)$ bound states of the Morse potential, $|j v\rangle$, which form a basis of the corresponding Hilbert space we can change to another basis, the CLM states $|j k(C L M)\rangle$, which is given by the following combination of the former,

$$
|j k(C L M)\rangle=N_{k}^{-1 / 2} \sum_{v=0}^{j-1} \mathcal{N}_{j v}^{-1 / 2} P_{j-1}^{(v)}\left(y_{k}\right)|j v\rangle
$$

where $y_{k}$ are, as before, the zeros of $L_{j}^{(1)}(y)$. The corresponding wave functions in coordinate space are

$$
\langle x \mid j k(C L M)\rangle=\Phi_{j k}(x)=N_{k}^{-1 / 2} y \exp \left[-\frac{y}{2}\right] Q_{j-1}^{(k)}(y),
$$

where $Q_{j-1}^{(k)}(y)$ is a $(j-1)$-degree polynomial defined by

$$
\begin{aligned}
Q_{j-1}^{(k)}(y) & =\sum_{v=0}^{j-1} \mathcal{N}_{j v}^{-1} P_{j-1}^{(v)}\left(y_{k}\right) P_{j-1}^{(v)}(y) \\
& =\sum_{n=0}^{j-1}(n+1)^{-1} L_{n}^{(1)}\left(y_{k}\right) L_{n}^{(1)}(y) .
\end{aligned}
$$

Using Eq. (10), we get

$$
Q_{j-1}^{(k)}(y)=L_{j-1}^{(1)}\left(y_{k}\right) \frac{L_{j}^{(1)}(y)}{y-y_{k}} .
$$

It is clear that $Q_{j-1}^{(k)}(y)$ vanishes when $y=y_{\ell}$ is a zero of $L_{j}^{(1)}(y)$ different from $y_{k}$. In Fig. 1 the wave functions $\Phi_{j k}(x)$ are plotted for the case of $j=5$. Each one of the five functions is localized mainly around one zero of the generalized Laguerre polynomial $L_{5}^{(1)}(y)$ and is null in the rest of its zeros.

These CLM states have the following properties.

(a) Orthogonality.

$$
\langle j k(\mathrm{CLM}) \mid j \ell(\mathrm{CLM})\rangle=\int d x \Phi_{j k}(x) \Phi_{j \ell}(x)=\delta_{k \ell} .
$$

(b) Zeros. $\Phi_{j k}(x)=0$ for $x=-\ln \left(y_{\ell} / 2 j+1\right)$, where $y_{\ell}$ is a zero of $L_{j}^{(1)}(y)$ different from $y_{k}$.

(c) Matrix elements of $y$. These can be easily evaluated from the explicit integration since $y$ can be rewritten as $y$ 


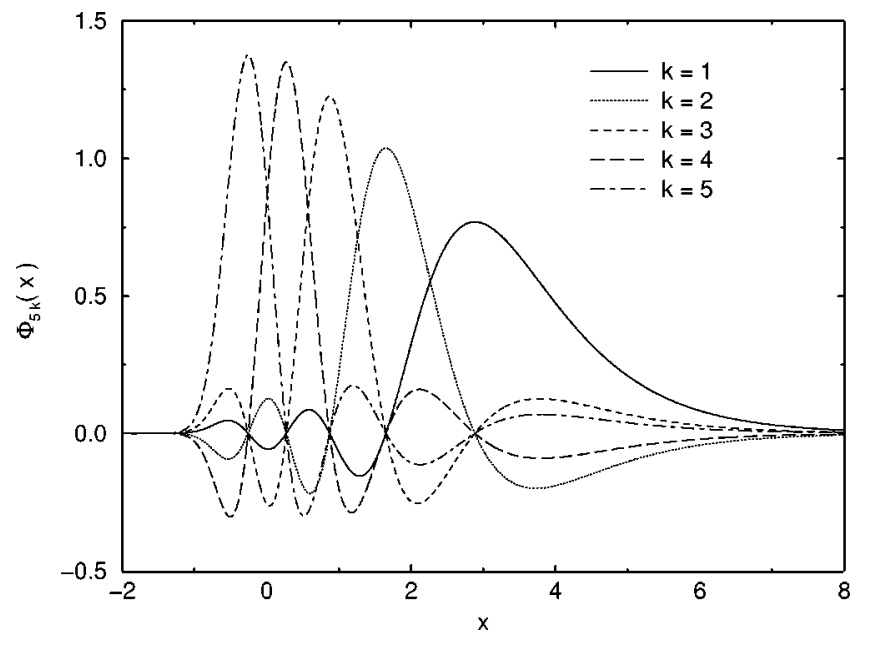

FIG. 1. Configuration localized Morse wave functions $\Phi_{j k}(x)$ for $j=5$.

$=y_{k}+\left(y-y_{k}\right)$. The contribution to the integral of the part $\left(y-y_{k}\right)$ is zero, since $\left(y-y_{k}\right) Q_{j-1}^{(k)}(y)$ is proportional to $L_{j}^{(1)}(y)[$ Eq. (18)] and its integral with any polynomial of degree less than $j$ vanishes. Thus, only the contribution coming from $y_{k}$ is left and the orthogonality of the CLM wave functions gives the final result

$$
\langle j k(\mathrm{CLM})|y| j \ell(\mathrm{CLM})\rangle=y_{k} \delta_{k \ell} .
$$

Thus, the CLM states are eigenstates of the Morse variable $y$ in the basis of the bound states.

(d) Matrix elements of $\left(y-y_{k}\right)\left(y-y_{\ell}\right)$. These can be easily evaluated using the previous expressions. The result is

$$
\begin{aligned}
\left\langle j k(\mathrm{CLM})\left|\left(y-y_{k}\right)\left(y-y_{\ell}\right)\right| j \ell(\mathrm{CLM})\right\rangle \\
=\frac{L_{j-1}^{(1)}\left(y_{k}\right)}{\sqrt{\left[L_{j-1}^{(1)}\left(y_{k}\right)\right]^{2}}} \frac{L_{j-1}^{(1)}\left(y_{\ell}\right)}{\sqrt{\left[L_{j-1}^{(1)}\left(y_{\ell}\right)\right]^{2}}} \sqrt{y_{k} y_{\ell}} \\
=\operatorname{sgn}\left[L_{j-1}^{(1)}\left(y_{k}\right)\right] \operatorname{sgn}\left[L_{j-1}^{(1)}\left(y_{\ell}\right)\right] \sqrt{y_{k} y_{\ell}} \\
=(-1)^{k-\ell} \sqrt{y_{k} y_{\ell}} .
\end{aligned}
$$

To obtain this last expression one has to realize that the zeros of the polynomials $L_{j-1}^{(1)}(y)$ alternate with those of $L_{j}^{(1)}(y)$. Thus, $L_{j-1}^{(1)}\left(y_{k}\right)$ evaluated at the zeros of $L_{j}^{(1)}(y)$ change sign as $(-1)^{k+1}$ if the usual standardization [8] is used.

(e) Matrix elements of $\left(y-y_{k}\right)\left(y-y_{\ell}\right)[y-(2 j+2)]$. These can be evaluated using the previous expressions, as well as the recurrence relations for the Laguerre polynomials, from which

$$
[y-(2 j+2)] L_{j}^{(1)}(y)=-(j+1)\left[L_{j-1}^{(1)}(y)+L_{j+1}^{(1)}(y)\right] .
$$

Using orthogonality, we get

$$
\left\langle j k(C L M)\left|\left(y-y_{k}\right)\left(y-y_{\ell}\right)[y-(2 j+2)]\right| j \ell(\mathrm{CLM})\right\rangle=0 .
$$

(f) Approximate expressions of the matrix elements of a generic function of $y /(2 j+1)$. If $F(y /(2 j+1)) \equiv$
TABLE I. Exact matrix elements of operator $x$ for Morse eigenstates. $j=5$.

\begin{tabular}{ccrrrr}
\hline \hline$v \backslash v^{\prime}$ & 0 & 1 & \multicolumn{1}{c}{2} & \multicolumn{1}{c}{3} & \multicolumn{1}{c}{4} \\
\hline 0 & 0.1461 & 0.3143 & -0.0722 & 0.0275 & -0.0129 \\
1 & & 0.4934 & 0.4666 & -0.1361 & 0.0582 \\
2 & & & 0.9596 & 0.6000 & -0.2004 \\
3 & & & & 1.6513 & 0.7127 \\
4 & & & & & 2.9251 \\
\hline \hline
\end{tabular}

$F(\exp (-x))$ is any continuous and derivable function of $y / 2 j+1$ in the region in which the zeros of $L_{j}^{(1)}(y)$ are located, then the approximate calculation of its diagonal and off-diagonal matrix elements can be evaluated by using a third order polynomial approximation. Using the results obtained above we get

$$
\begin{aligned}
& \left\langle j k(\mathrm{CLM})\left|F\left(\frac{y}{2 j+1}\right)\right| j \ell(\mathrm{CLM})\right\rangle \\
& \quad \simeq F\left(\frac{y_{k}}{2 j+1}\right) \delta_{k \ell}+\frac{1}{2}(-1)^{k-\ell} \frac{\sqrt{y_{k} y_{\ell}}}{(2 j+1)^{2}} F^{\prime \prime}\left(\frac{y_{0}}{2 j+1}\right),
\end{aligned}
$$

where $y_{0}=\left[y_{k}+y_{\ell}+(2 j+2)\right] / 3$. For large $j$ the term containing $F^{\prime \prime}(y /(2 j+1))$ can be neglected compared to the other one. Thus the matrix elements of any function of $y$ (or $x$ ) between CLM states are, to a good approximation, just the values of the function evaluated in the corresponding zeros of the polynomial $L_{j}^{(1)}(y)$.

(g) Approximate expressions of the matrix elements of a function $f(r)$ in a basis of Morse eigenstates. Any function of $r=r_{e}+(x / \beta)$ is also a function of $y /(2 j+1)$, as $r=r_{e}$ $-(1 / \beta) \ln [y / 2 j+1]$. Using Eq. (24), and ignoring the term in $F^{\prime \prime}$, one gets

$$
\left\langle j v|f(r)| j v^{\prime}\right\rangle \simeq \sum_{k}\langle j v \mid j k(C L M)\rangle f\left(r_{k}\right)\left\langle j k(C L M) \mid j v^{\prime}\right\rangle
$$

where $r_{k}=r_{e}-(1 / \beta) \ln \left[y_{k} / 2 j+1\right]$ is determined by the zeros, $y_{k}$, of the Laguerre polynomial $L_{j}^{(1)}(y)$, as well as by the equilibrium position $r_{e}$, the size parameter $\beta$, and the parameter $j$.

The accuracy of Eq. (25) will be better for larger $j$, and also when $f(r)$ is a smooth function of $r$ close to $r_{e}$. Besides, we might expect that the oscillating nature of the $F^{\prime \prime}$ correction in Eq. (24) due to the factor $(-1)^{k-\ell}$ will tend to cancel the corrections evaluated for the lower Morse eigenstates, which have few nodes.

In order to test how good the approximations done are, in Tables I and II we show the matrix elements of the operator $x$ for the case $j=5$. In Table I the eigenstates of the Morse potential are used and exact integration is performed while in Table II Eq. (25) is used. It can be observed that even for this very unfavorable case of small $j$ the agreement is good, especially for the lowest states in the potential. The agreement improves greatly when $j$ increases. In Fig. 2 matrix elements of the operator $x$ are shown for the value $j=22$. They are 
TABLE II. Approximate matrix elements of operator $x$ using CLM states. $j=5$.

\begin{tabular}{cccrrr}
\hline \hline$v \backslash v^{\prime}$ & 0 & 1 & \multicolumn{1}{c}{2} & \multicolumn{1}{c}{3} & \multicolumn{1}{c}{4} \\
\hline 0 & 0.1461 & 0.3146 & -0.0730 & 0.0290 & -0.0146 \\
1 & & 0.4920 & 0.4706 & -0.1440 & 0.0683 \\
2 & & & 0.9468 & 0.6299 & -0.2453 \\
3 & & & & 1.5646 & 0.8886 \\
4 & & & & & 2.2608 \\
\hline \hline
\end{tabular}

evaluated exactly by direct integration using the Morse wave functions (symbols) and approximately by using the CLM states (lines). Three different cases $\left(v_{f}=v_{i}, v_{f}=v_{i}+1\right.$, and $\left.v_{f}=v_{i}+2\right)$ are presented as a function of $v_{i}$. Agreement is seen to be good and deviations only occur when we are approaching very close to the dissociation limit. Note that in the harmonic limit all the transitions with $\Delta v \neq 1$ would vanish. The agreement for $\Delta v=0,2$ indicates that the CLM states are well suited to describe anharmonic effects.

\section{APPLICATIONS TO THE DIATOMIC MOLECULES HF AND DF}

In this section we will show how to exploit some of the possibilities of the CLM wave functions by studying vibrational dipole matrix elements in the diatomic molecules HF and DF. For these molecules a rather complete experimental information exists [9]. Within the Born-Oppenheimer approximation, the vibrational transitions for the electronic ground state band are determined by the matrix elements,

$$
R_{v}^{v^{\prime}}=\int_{0}^{\infty} \Psi_{v}^{*}(r) \mu(r) \Psi_{v^{\prime}}(r) r^{2} d r
$$

The vibrational wave functions $\Psi_{v}(r)$ are calculated by solving the Schrödinger equation for the interatomic poten-

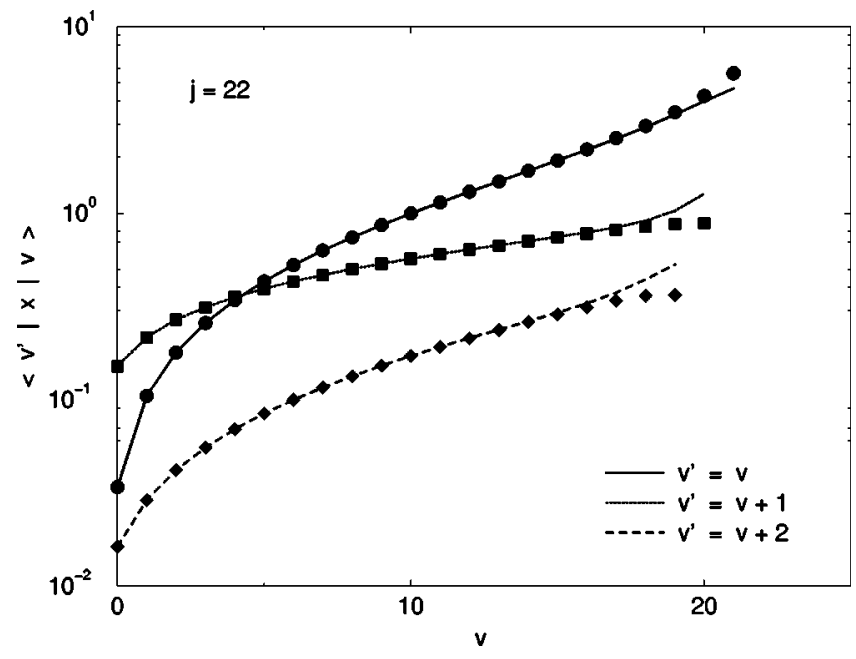

FIG. 2. Matrix elements of the operator $x$ for Morse vibrational states as a function of $v_{i}$, for $j=22$. Symbols correspond to the exact integration using Morse wave functions. Lines correspond to the approximate analytic expression derived from CLM states. tial energy function [10]. The dipole moment function in a diatomic molecule is given as a function of the interatomic separation $r$ by

$$
\mu(r)=\left\langle\Psi_{g}^{e}(r)\left|\sum_{i} e_{i} z_{i}\right| \Psi_{g}^{e}(r)\right\rangle
$$

where $\left|\Psi_{g}^{e}(r)\right\rangle$ is the electronic wave function for the lowest electronic state, the sum on $i$ runs over all electrons and nuclei, $e_{i}$ are their charges, and $z_{i}$ are the projections of the their coordinates along the interatomic direction. The dipole moment function can be determined in principle from $a b$ initio calculations. However, it is found to be very sensitive to the choice of basis functions. Hence it has been obtained by fitting experimental data of vibrational transitions [9].

We will use the properties of the CLM states to obtain closed expressions for the vibrational matrix elements $R_{v}^{v^{\prime}}$ as a function of the dipole moment function. Let us assume that the Morse potential is a good representation of the interatomic interaction. Hence, we substitute the vibrational wave functions $\Psi_{v}(r)$ by Morse wave functions. Then one has to evaluate the following integral:

$$
R_{v}^{v^{\prime}}(\text { Morse })=\int_{-\infty}^{\infty} \Psi_{j v}^{*}(x) \mu(r) \Psi_{j v^{\prime}}(x) d x
$$

where $x=\beta\left(r-r_{e}\right)$ and $\Psi_{j v}(x)$ are Morse wave functions [Eq. (5)]. As the dipole moment function is a smoothly varying function of $x$, and hence also of the Morse variable $y$, Eq. (25) can be used as an approximation to the evaluation of Eq. (28),

$$
R_{v}^{v^{\prime}}(\mathrm{CLM})=\sum_{k}\langle j v \mid j k(\mathrm{CLM})\rangle \mu\left(r_{k}\right)\left\langle j k(\mathrm{CLM}) \mid j v^{\prime}\right\rangle .
$$

This expression only requires the knowledge of the value of the dipole moment at certain points $\left(r_{k}\right)$, determined by the zeros of the appropriate generalized Laguerre polynomial, and the overlaps $\langle j v \mid j k(\mathrm{CLM})\rangle$. These have simple analytical forms that are obtained directly from the definition of the CLM states [Eq. (15)].

With the information available from spectroscopic data, the values of $r_{e}=0.9172 \AA, j=22$ and $\beta=2.261 \AA^{-1}$ for HF and $r_{e}=0.9170 \AA, j=32$ and $\beta=2.222 \AA^{-1}$ for DF are obtained. In Fig. 3 the dipole moment functions for HF and DF are plotted as given in Ref. [9], up to $1.7 \AA$, and it is linearly extrapolated beyond. Full squares correspond to the values of $r_{k}$ determined from the zeros of the generalized Laguerre polynomial $L_{22}^{(1)}(y)$, which is the relevant one for HF. Open triangles correspond to the values of $r_{k}$ determined from the zeros of the generalized Laguerre polynomial $L_{32}^{(1)}(y)$ which is the relevant one for DF. Since some zeros of $L_{22}^{(1)}(y)$ [and $\left.L_{32}^{(1)}(y)\right]$ correspond to interatomic separations larger than $1.7 \AA$, a simple linear extrapolation has been done. We have checked that the results presented below are not sensitive to a different reasonable extrapolation (exponential, Gaussian, ... ).

In Figs. 4 and 5 vibrational dipole matrix elements for $\mathrm{HF}$ and DF are presented as a function of $v_{i}$. The results ob- 


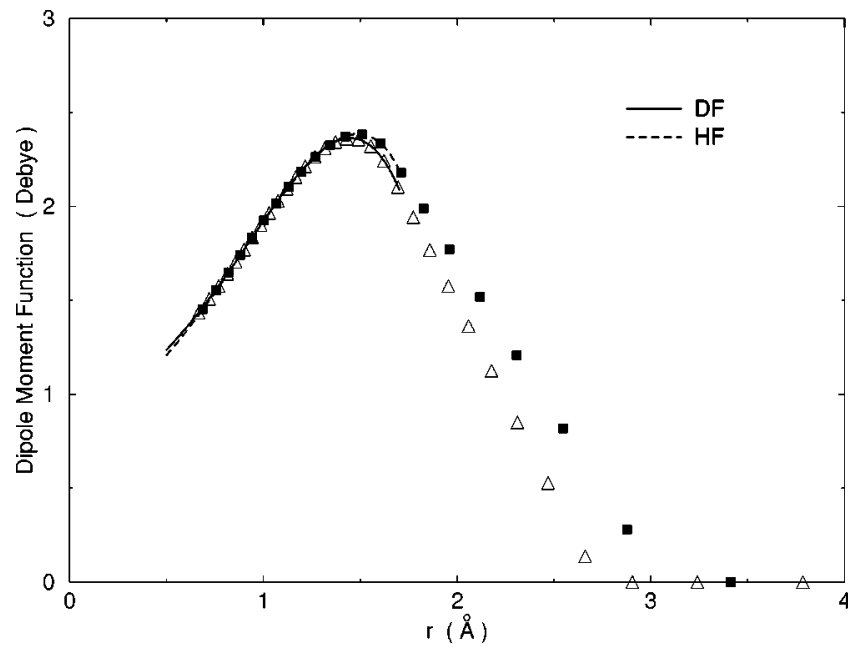

FIG. 3. Dipole moment function versus internuclear separation for $\mathrm{HF}$ and $\mathrm{DF}$. The dipole moment function up to internuclear separations of $1.7 \AA$ is taken from Ref. [9] and a linear extrapolation for larger values has been done (see text). Full squares correspond to the zeros of the generalized Laguerre polynomial $L_{22}^{(1)}(y)$. Open triangles correspond to the zeros of the generalized Laguerre polynomial $L_{32}^{(1)}(y)$.

tained in [9] using Eq. (26) are compared with our Eq. (29). Note that not only the matrix elements with $\Delta v=1$, dominant in the harmonic limit, are well reproduced, but also the terms with $\Delta v=0,2$. Besides, the transitions to the vibrational ground state $v_{f}=0$, which change over several orders of magnitude, are well reproduced. The agreement is remarkable, considering that Eq. (26) implies the calculation of vibrational wave functions solving the Schrödinger equation, as well as performing the radial integration with the dipole moment function, while Eq. (29) is just a sum of the dipole moment function evaluated at certain points, multiplied by some analytic coefficients. It is worth noting that all the computed matrix elements have the same signs as written in Ref. [9] except for the transitions from the ground state to the state with $v=6$ in both $\mathrm{HF}$ and $\mathrm{DF}$, for which also the magnitudes are rather different. This is due to the fact that the

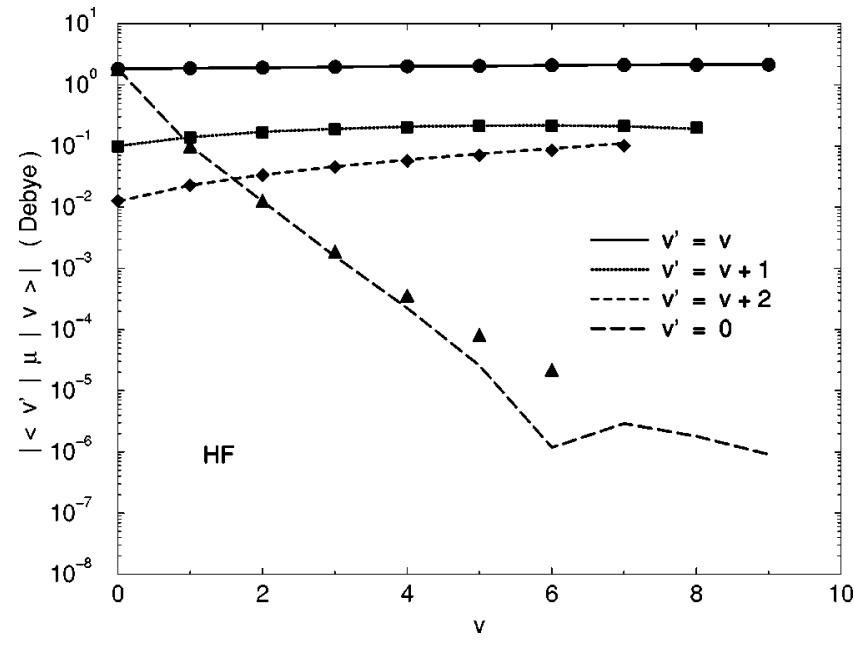

FIG. 4. Dipole matrix elements for the vibrational states of $\mathrm{HF}$ as a function of $v_{i}$. Symbols correspond to Ref. [9]. Lines correspond to the analytic expression derived from CLM states.

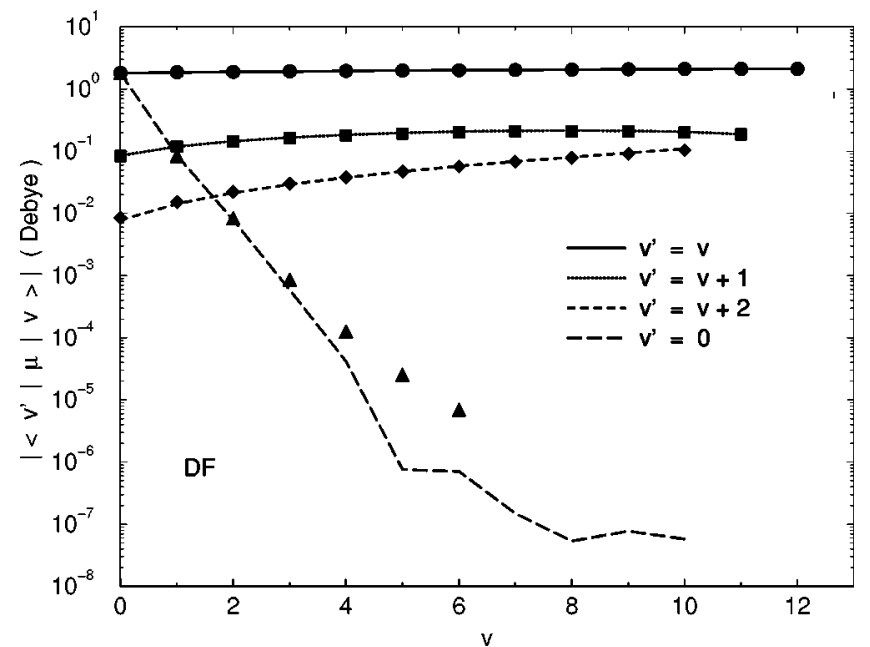

FIG. 5. Dipole matrix elements for the vibrational states of DF. The notation is the same as in Fig. 4.

vibrational wave functions in Ref. [9] are not exactly Morse wave functions and so it is not surprising that in the evaluation of the matrix elements $\langle 0|\mu| 6\rangle$, for which delicate cancelations occur, the present work and Ref. [9] give different results.

\section{OUTLOOK}

The use of CLM states to evaluate matrix elements is closely related, from the mathematical point of view, to the approximate evaluation of integrals by using quadratures [8]. In particular, we have derived the expression

$$
\int_{0}^{\infty} d x x \exp [-x] f(x) \simeq \sum_{k=1}^{j} w(j, k) f\left(x_{k}\right),
$$

where $x_{k}$ are the zeros of $L_{j}^{(1)}(x)$, and $w(j, k)=N_{k}^{-1}$ [see Eq. (13)]. This expression, which is very accurate [the error is of the order of the $2 j$ derivative of the function $f(x)]$, can be used to evaluate, for example, Franck-Condon factors, which are proportional to overlap integrals of vibrational wave functions corresponding to different potential wells. This work is in progress.

The usefulness of configuration localized states is not restricted to Morse wave functions. Previously [11,12], a similar procedure was used for studying rotational states in nuclei, giving rise to states with definite orientation in space, that was determined by the zeros of Legendre polynomials [11] for a $K=0$ band, and the zeros of other families of orthogonal polynomials for $K \neq 0$ bands [12]. These states were very useful to describe the effect of quadrupole coupling in the collisions of deformed nuclei. Coming back to molecular physics, the method presented here can be extended to other potentials of interest in this field as it is the Pöschl-Teller potential.

Although in this paper the simple case of diatomic molecules has been treated to illustrate the usefulness of the CLM states, this method can be applied to polyatomic molecules. In that case the dipole operator is a function of all relevant interatomic separations $r_{i j}$. Thus, its matrix elements are approximately diagonal in a basis obtained as the 
tensor product of the CLM wave functions for all separations $r_{i j}$. If the vibrational wave functions for the polyatomic molecule are expanded in terms of product of local Morse wave functions corresponding to $r_{i j}$, then the evaluation of the dipole matrix elements can done analytically. Indeed, the coefficients of the expansion in terms of the Morse local basis can be obtained from recent algebraic treatments of molecular vibrational structure within the su(2) model [13].

In the context of the su(2) algebraic models the calculation of dipole vibrational transitions is a complex problem since the algebraic expression of the dipole operator in terms of generators of the relevant algebra is not known. In Ref. [14], the matrix elements of the dipole operator are parametrized with a suitable function and the parameters are fitted to reproduce the experimental data. The CLM method can be used as a hybrid model in which, from the knowledge of the dipole moment function $\mu(r)$, obtained from ab initio calculations or using experimental information, one can obtain an operator

$$
\hat{\mu}=\sum_{k}|j k(\mathrm{CLM})\rangle \mu\left(r_{k}\right)\langle j k(\mathrm{CLM})|
$$

suitable for an algebraic approach.

The CLM states provide us also with a scheme to relate the description of operators in terms of coordinates and in terms of an expansion of the generators of the dynamical algebra. For the Morse potential, an algebraic treatment based in the su(2) group will describe all operators as an expansion in terms of powers of the generators $\hat{J}_{i}, i=0, \pm$. Thus,

$$
\hat{O}^{a l g}=c_{0}+\sum_{i} c_{i} \hat{J}_{i}+\sum_{i j} c_{i j} \hat{J}_{i} \hat{J}_{j}+\cdots .
$$

In terms of coordinates, a momentum-independent operator is given by a function $O(r)$. However, we can approximate

$$
O(r) \simeq \sum_{k}|j k(\mathrm{CLM})\rangle O\left(r_{k}\right)\langle j k(\mathrm{CLM})| .
$$

Thus, a mapping between both expressions for the operators can be achieved. The coordinate representation of an algebraic operator will be given by

$$
O\left(r_{k}\right)=\left\langle j k(\mathrm{CLM})\left|\hat{O}^{a l g}\right| j k(\mathrm{CLM})\right\rangle .
$$

Note that this gives the function $O(r)$ at certain points $r$ $=r_{k}$. The function at other values of $r$ could be obtained by interpolation and extrapolation. The algebraic representation of a coordinate operator can be obtained by fitting the parameters in the expansion of $\hat{O}^{a l g}$ so that the matrix elements of $O(r)$ are reproduced.

\section{SUMMARY AND CONCLUSIONS}

In this paper the configuration localized Morse (CLM) states are introduced and their properties are analyzed. The CLM states are the eigenfunctions of the Morse variable in the basis generated by the bound states of the Morse potential. The CLM states are analytically determined in terms of the zeros of the polynomial $L_{j}^{(1)}(y)$. They are strongly localized in configuration space, providing us with a powerful tool to calculate analytically, to a good approximation, matrix elements of arbitrary functions of the coordinate between Morse wave functions.

Using the properties of CLM, the matrix elements between vibrational states of the dipole operator for a diatomic molecule can be calculated. The necessary input, that may be obtained from the vibrational spectrum, is the dipole moment function $\mu(r)$, the equilibrium separation $r_{e}$, and the parameters $j$ and $\beta$. Then, the matrix elements are given by a linear combination of the dipole moment function evaluated at certain separations that are determined by the zeros of $L_{j}^{(1)}(y)$. It should be noticed that in this method the vibrational wave functions do not have to be explicitly calculated, nor does the radial integration have to be performed. This method has been applied to the calculation of vibrational transitions in HF and DF giving very similar results to conventional calculations where realistic vibrational wave functions are used [9].

We consider that the CLM states constitute a useful starting point in order to obtain analytical approximations for different magnitudes, such as vibrational transitions intensities and Franck-Condon factors. It would be very interesting to extend their application to polyatomic molecules, where the simplicity of the associated expressions can be essential. Besides, the CLM states could provide us with a connection between algebraic models and coordinate descriptions of molecular properties.

\section{ACKNOWLEDGMENTS}

This work was supported in part by the Spanish DGICYT under Project No. PB95-0533 and by the ISC program of the European Commission under Project No. CI1*-CT94-0072. We acknowledge useful discussions with F. Pérez-Bernal, A. Frank, and R. Lemus.
[1] P. M. Morse, Phys. Rev. 34, 57 (1929).

[2] M. Berrondo, A. Palma, and J. L. López-Bonilla, Int. J. Quantum Chem. XXXI, 243 (1987).

[3] J. N. Huffaker and Loc Binh Tran, J. Chem. Phys. 76, 3838 (1982).

[4] M. L. Sage, Chem. Phys. 35, 375 (1978).

[5] M. J. Englefield and C. Quesne, J. Phys. A 24, 3557 (1991).
[6] A. López Pĩnero and B. Moreno, Phys. Rev. A 38, 5847 (1988).

[7] M. Berrondo and A. Palma, J. Phys. A 13, 773 (1980).

[8] Handbook of Mathematical Functions, edited by M. Abramowitz and I. Stegun (Dover, New York, 1972).

[9] R. N. Sileo and T. A. Cool, J. Chem. Phys. 65, 117 (1976). 
[10] R. E. Meredith and F. G. Smith, J. Quant. Spectrosc. Radiat. Transf. 13, 89 (1973).

[11] M. A. Nagarajan, B. Balantekin, and N. Takigawa, Phys. Rev. C 34, 894 (1986).

[12] M. V. Andres, J. Gomez-Camacho, and M. A. Nagarajan,
Phys. Rev. C 45, 1339 (1992).

[13] A. Frank, R. Lemus, R. Bijker, F. Pérez-Bernal, and J. M. Arias, Ann. Phys. (N.Y.) 252, 211 (1996).

[14] F. Iachello, A. Leviatan, and A. Mengoni, J. Chem. Phys. 95, 1449 (1990). 